\title{
A Remark on the Transformations between Different Representations
}

\section{Zhang Yue}

College of Physics and Information Science, Hunan Normal University, Changsha 410081, China Email: phys_zhangyue@126.com

\begin{abstract}
:
The paper respectively studies the transformations of spin component operators and their eigenfunctions from $S_{z}$ to $S_{x}$ representation. The studies demonstrate that if using the matrix of obtaining from the equation of transforming the eigenfunctions of $\hat{S}_{x}$ and in terms of the transformation arising from Ref. [7] for all of the spin component operators from $S_{z}$ to $S_{x}$ representation, a negative symbol will occur in the result, which is not concordant with the commutation relation between $\hat{S}_{x}$ and $\hat{S}_{z}$. Moreover, this matrix of transformation can not be applied to the transformation of the eigenfunctions of $\hat{S}_{z}$ or $\hat{S}_{y}$. It therefore concludes that with respect to the transformation of representation of an operator and its eigenfunctions, the matrix of transformation should be obtained from the equation of transforming the eigenfunctions of the same operator from the old to the new representation.
\end{abstract}

\section{Keywords:}

transformation of representation; mathematical definition; matrix of transformation; old representation; new representation

\section{Introduction}

In quantum mechanics, the theory about the representation of state vector (or wave function) and the operator as well as the transformation between different representations is an important subject which attracts the remarkable interesting of a lot of researchers ${ }^{[1-4]}$. There are two equivalent mathematical expressions for the transformation of representations, if $\hat{F}$ is an operator of representing a physical quantity, $U$ is a linear operator, the reverse matrix of the matrix of $U$ exists for all of the wave functions, thus, the definition of the transformation of representations for the operator $\hat{F}$ and its eigenfunctions from $\mathrm{B}$ to $\mathrm{A}$ representation is ${ }^{[5-6]}$ :

$$
\psi_{A}=U^{-1} \psi_{B}, \quad F_{A}=U^{-1} F_{B} U,
$$

where $F_{A}\left(F_{B}\right)$ is the matrix of $\hat{F}$ in $A(B)$ representation, $\psi_{A}\left(\psi_{B}\right)$ notes the eigenfunction of $\hat{F}$ in $A(B)$ representation. It can be proven that the transformation of representation is also an unitary transformation ${ }^{[5-6]}$, thus, $U U^{-1}=U^{-1} U=I$, multiplying the transformation formula of operator in eq. (1) with $U$ from the left and with $U^{-1}$ from the right, moreover, multiplying the transformation of formula of eigenfunction in eq. (1) with $U$ from the left it arrives

$$
\psi_{B}=U \psi_{A}, \quad F_{B}=U F_{A} U^{-1}
$$


Eq.(2) is equivalent of eq.(1), it indicates the transformation of $\hat{F}$ and its eigenfunctions from A to B representation, of course, A or B representation can be replaced by the representation of $\hat{F}$ itself.

Concerning the application of the transformation of representation, the teaching materials generally employ eq. $(1)^{[5-6]}$, but there are some researchers like to employ eq.(2) ${ }^{[7-8]}$. The key problem for the transformation of representation is how to determine its transformation matrix, if some mistakes or puzzles occurring in the transformation result, these are usually caused by incorrect transformation matrix employed. Taking the electronic spin component operators and their eigenfunctions as an example, the paper will present some discussions and studies by use of eq.(1).

\section{Review of Literature}

\section{Transformation of Operator}

A typical application is the transformation of electronic spin operators $\hat{S}_{i}(i=x, y, z)$ from $S_{z}$ (old representation) to $S_{x}$ (new representation) representation. In $S_{z}$ representation, from the commutation relations between $\hat{S}_{x}$ and $\hat{S}_{y}$; or $\hat{S}_{x}$ and $\hat{S}_{z}$; or $\hat{S}_{y}$ and $\hat{S}_{z}$, it can be derived that the spin operators are written:

$$
S_{x}=\frac{\mathrm{h}}{4 \pi}\left(\begin{array}{ll}
0 & 1 \\
1 & 0
\end{array}\right), \quad S_{y}=\frac{\mathrm{h}}{4 \pi}\left(\begin{array}{cc}
0 & -i \\
i & 0
\end{array}\right), \quad S_{z}=\frac{\mathrm{h}}{4 \pi}\left(\begin{array}{cc}
1 & 0 \\
0 & -1
\end{array}\right) .
$$

With respect to the transformation of representation of operators, some people obtained the transforming matrix from the transforming formula of the eigenfunctions of one of the spin operators from $S_{z}$ to $S_{x}$ representation ${ }^{[7]}$. For example, using the matrix of $S_{x}$ in eq. (3) to solve the equations of its eigenvalues, it obtains the eigenfunctions of $\hat{S}_{x}$ in $S_{z}$ representation

$$
\chi_{1 / 2}\left(S_{x}\right)=\frac{1}{\sqrt{2}}\left(\begin{array}{l}
1 \\
1
\end{array}\right), \quad \chi_{-1 / 2}\left(S_{x}\right)=\frac{1}{\sqrt{2}}\left(\begin{array}{c}
-1 \\
1
\end{array}\right)
$$

where the subscripts $1 / 2$ and $-1 / 2$ of $\chi$ respectively correspond to the eigenvalues $\frac{h}{4 \pi}$ and $-\frac{h}{4 \pi}$. According to the assumption of electronic spin ${ }^{[6]}$, the eigenfunctions of $\hat{S}_{x}$ (using $S_{x}^{\prime}$ notes its matrix in $S_{x}$ representation) in $S_{x}$ representation are written

$$
\chi_{1 / 2}\left(S_{x}^{\prime}\right)=\left(\begin{array}{l}
1 \\
0
\end{array}\right), \quad \chi_{-1 / 2}\left(S_{x}^{\prime}\right)=\left(\begin{array}{l}
0 \\
1
\end{array}\right)
$$


If using eq.(1) to perform the transformation, thus

$$
\chi_{1 / 2}\left(S_{x}^{\prime}\right)=U^{-1} \frac{1}{\sqrt{2}}\left(\begin{array}{l}
1 \\
1
\end{array}\right)=\left(\begin{array}{l}
1 \\
0
\end{array}\right), \quad \chi_{-1 / 2}\left(S_{x}^{\prime}\right)=U^{-1} \frac{1}{\sqrt{2}}\left(\begin{array}{c}
-1 \\
1
\end{array}\right)=\left(\begin{array}{l}
0 \\
1
\end{array}\right)
$$

it calculates out from eq.(6) that

$$
U^{-1}=\frac{1}{\sqrt{2}}\left(\begin{array}{cc}
1 & 1 \\
-1 & 1
\end{array}\right), \quad U=\frac{1}{\sqrt{2}}\left(\begin{array}{cc}
1 & -1 \\
1 & 1
\end{array}\right) .
$$

Obviously, $U U^{-1}=U^{-1} U=I$.

It is easy to prove that the transformation of representation does not change the commutation relations between $\hat{S}_{x}$ and $\hat{S}_{y}$; or $\hat{S}_{x}$ and $\hat{S}_{z}$; or $\hat{S}_{y}$ and $\hat{S}_{z}$. Using eq.(1) and eq.(3), the transformation results of spin operator from $S_{z}$ to $S_{x}$ representation are given by ${ }^{[7]}$

$$
\begin{gathered}
S_{x}^{\prime}=U^{-1} S_{x} U=\frac{\mathrm{h}}{4 \pi}\left(\begin{array}{cc}
1 & 0 \\
0 & -1
\end{array}\right), \quad S_{y}^{\prime}=U^{-1} S_{y} U=\frac{\mathrm{h}}{4 \pi}\left(\begin{array}{cc}
0 & -i \\
i & 0
\end{array}\right), \\
S_{z}^{\prime}=U^{-1} S_{z} U=-\frac{\mathrm{h}}{4 \pi}\left(\begin{array}{ll}
0 & 1 \\
1 & 0
\end{array}\right) .
\end{gathered}
$$

According to the commutation relation between $\hat{S}_{x}$ and $\hat{S}_{z}$, the transformation result of eq. (8) should be just to exchange the matrix of $S_{z}$ with that of $S_{x}$ in eq.(3) ${ }^{[8]}$, but a negative symbol occurs in $S_{z}^{\prime}$ of eq.(8). The negative symbol of the transformation results in Ref.[7] occurs in the matrix of $S_{y}^{\prime}$, it should be a special case, because the reverse matrix of its transformation matrix is just as same as itself.

\section{Discussion}

\subsection{Transformation of Eigenfunctions}

If similar to the transformation of representation of operator eq.(8), using $U^{-1}$ of eq. (7) to transform the eigenfunctions of $\hat{S}_{z}$ from $S_{z}$ to $S_{x}$ representation, it arrives

$$
\chi_{1 / 2}\left(S_{z}^{\prime}\right)=U^{-1}\left(\begin{array}{l}
1 \\
0
\end{array}\right)=\frac{1}{\sqrt{2}}\left(\begin{array}{c}
1 \\
-1
\end{array}\right), \quad \chi_{-1 / 2}\left(S_{z}^{\prime}\right)=U^{-1}\left(\begin{array}{l}
0 \\
1
\end{array}\right)=\frac{1}{\sqrt{2}}\left(\begin{array}{l}
1 \\
1
\end{array}\right)
$$

Because in $S_{x}$ representation, the matrix expressions of $\hat{S}_{z}$ and $\hat{S}_{x}$ should be the same as just exchanging their matrix expressions in $S_{z}$ representation, therefore, from the equation of eigenvalue, it obtains that the eigenfunctions of $\hat{S}_{z}$ in $S_{x}$ is just the same as eq.(4), the results of eq.(9) are evidently wrong. This demonstrates that the eigenfunctions of spin component operators can not be transformed from $S_{z}$ to $S_{x}$ representation by use of eq.(7) 
That the reason of the mistakes occurs in eq.(9) is due to the incorrect transformation matrix employed. The eigenfunctions of $\hat{S}_{z}$ in the representation of itself are the totally same as eq.(5), using eq.(4) and eq.(5), the transformation of representation for the eigenfunctions of $\hat{S}_{z}$ from $S_{z}$ to $S_{x}$ representation are given by

$$
\chi_{1 / 2}\left(S_{z}^{\prime}\right)=U^{-1}\left(\begin{array}{l}
1 \\
0
\end{array}\right)=\frac{1}{\sqrt{2}}\left(\begin{array}{l}
1 \\
1
\end{array}\right), \quad \chi_{-1 / 2}\left(S_{z}^{\prime}\right)=U^{-1}\left(\begin{array}{l}
0 \\
1
\end{array}\right)=\frac{1}{\sqrt{2}}\left(\begin{array}{c}
-1 \\
1
\end{array}\right)
$$

from eq.(10) it calculates out

$$
U^{-1}=\frac{1}{\sqrt{2}}\left(\begin{array}{cc}
1 & -1 \\
1 & 1
\end{array}\right), \quad U=\frac{1}{\sqrt{2}}\left(\begin{array}{cc}
1 & 1 \\
-1 & 1
\end{array}\right)
$$

Therefore, with respect to the transformation of eq.(9), we must take the $U^{-1}$ of eq.(11) as the transformation matrix, it is just the reverse matrix of the $U^{-1}$ of eq.(7).

Thus, if using eq.(11) for the transformation of $\hat{S}_{z}$ from $S_{z}$ to $S_{x}$ representation, it obtains

$$
S_{z}^{\prime}=U^{-1} S_{z} U=\frac{1}{\sqrt{2}}\left(\begin{array}{ll}
1 & 1 \\
1 & 1
\end{array}\right) \frac{\mathrm{h}}{4 \pi}\left(\begin{array}{ll}
1 & 0 \\
0 & 1
\end{array}\right) \frac{1}{\sqrt{2}}\left(\begin{array}{ll}
1 & 1 \\
1 & 1
\end{array}\right)=\frac{\mathrm{h}}{4 \pi}\left(\begin{array}{ll}
0 & 1 \\
1 & 0
\end{array}\right)
$$

there is evidently no the negative symbol as in the matrix of $S_{z}^{\prime}$ of eq.(8) in this result of transformation.

According to the above discussions, we get a new recognition about the transformation of representation of spin operator eq.(8). if the transformation matrix for every spin component operator of eq. (3) is determined by the transformation formula of the eigenfunctions of itself, from the calculations it can be found that the result of the transformation is just to exchange the matrix of $S_{x}$ with that of $S_{z}$ in eq.(3), it does not generate the negative symbol in the matrix of $S_{z}^{\prime}$ of eq.(8).

Because the commutation relations between $\hat{S}_{x}$ and $\hat{S}_{y}$; or $\hat{S}_{x}$ and $\hat{S}_{z}$; or $\hat{S}_{y}$ and $\hat{S}_{z}$ are symmetrical from each other, therefore, it can similarly discuss the transformations of other spin component operators and their eigenfunctions from $S_{y}$ to $S_{x}$ representation, or from $S_{y}$ to $S_{z}$ representation. 


\section{Conclusion}

This paper respectively studied the transformation of the spin component operators and their eigenfunctions from $S_{z}$ to $S_{x}$ representation, and obtained the following conclusions:

1. With respect to the transformation of representation for every spin component operator and its eigenfuntions (or state vectors), the transformation matrix must be determined by the transformation formula of its eigenfunctions from the old to the new representation.

2. In consideration of the transformation of eq.(3) from $S_{z}$ to $S_{x}$ representation, if the transformation matrix for every spin component operator is determined by the transformation formula of its eigenfunctions, for example, the transformation matrix for $S_{x}$ employs eq.(7); the transformation matrix for $S_{z}$ employs eq.(11), the result of the transformation is just equal to exchange the matrix of $S_{x}$ with that of $S_{z}$ of eq.(3), it does not occur the negative symbol of $S_{z}^{\prime}$ of eq.(8), and it is consistent with the commutation relation between $\hat{S}_{x}$ and $\hat{S}_{z}$.

3. If the commutation relations of the component operators of other operator are similar to the commutation relations between the spin component operators, the conclusions (1) and (2) are applicable to the transformation of representation for the component operators of this operator and their eigenfunctions.

\section{References}

[1] Ohanian H C. Principles of quantum mechanics [M]. Englewood Cliffs: Prentice - Hall, 1990, 139-146.

[2] M Moshinsky and T H Seligman. Canonical transformation to action angle variables and their representation in quantum mechanics [J. Annals of Physics, 1978, 114 (2) : 243272.

[3] Joshua Wilkie and Paul Brumer. Transformation theory for phase-space representation of quantum mechanics []. Phys. Rev. A, 2000, 61:064101.

[4] P A Mello and M Moshinsky. Nonlinear canonical transformations and their representations in quantum mechanics D]. Journal of Mathematical Physics, 1975, 16 (10): 2017.

[5] A. S Davydov. Quantum Mechanics (Second Edition, Translated by D Ter Haar from Russian) [M]. Oxford: Pergamon Press, 1976, 117-121.

[6] Zhou Shi-Xun. The Course of Quantum Mechanics [M] (in Chinese). Beijing: Advanced Education Press, 1990, 114-119.

[7] Ka Xing-Lin. Representation transformation of Spin Operators and the Phase Factor D]. College Physics (in Chinese), 1988, 7(4):21-26.

[8] Dirac P A M. The Principles of Quantum Mechanics (Te 4th edition of translation in Chinese) [M]. Beijing: Scientific Press, 1979, 105-109. 